\title{
Effective transport properties of 3D multi-component microstructures with interface resistance
}

\author{
Denis Roussel ${ }^{\mathrm{a}, *}$, Aaron Lichtner ${ }^{\mathrm{b}}$, David Jauffrès ${ }^{\mathrm{a}}$, Rajendra K. Bordia ${ }^{\mathrm{c}}$, Christophe L. Martin ${ }^{\mathrm{a}, \mathrm{1}}$ \\ ${ }^{a}$ Univ. Grenoble Alpes, CNRS, SIMAP, F-38000 Grenoble, France \\ ${ }^{\mathrm{b}}$ Department of Materials Science and Engineering, University of Washington, Seattle, WA 98105, USA \\ ${ }^{\mathrm{c}}$ Department of Materials Science and Engineering, University of Clemson, SC 29634, USA
}

\begin{abstract}
A three-dimensional image analysis tool has been developed to compute the effective conductivity of a particulate composite made of pores (material 0 ) and two materials (1) and (2). Three types of resistances are considered: the two resistances between materials (1)-(1) and (2)-(2) and the charge transfer resis-tance at the interface between materials (1) and (2). The numerical tool uses voxelised 3D numerical microstructures generated by discrete element simulations to mimic the particulate nature of the tested material. The method is validated by comparing its results to a resistance network model that incorpo-rates interface resistance. The validation is conducted for both homogeneous and composite materials. Our tool has the advantage, compared with a resistance-network model, of allowing 3D tomography images to be treated. The method is applied to a microstructure typical of a composite Solid Oxide Fuel Cell cathode, however it is general enough to be a versatile tool for computing effective transport prop-erties of any composite material where interface properties must be taken into account.
\end{abstract}

\section{Introduction}

Synchrotron X-ray nano-tomography has become a powerful instrument for in situ understanding of material processing [1]. Focused Ion Beam (FIB) coupled with SEM and X-ray imaging techniques also provide direct visualization of the internal structure of materials. Using X-ray optics for magnification, spatial resolutions of up to $15 \mathrm{~nm}$ have been obtained by synchrotron radiation [2]. As these techniques improve in resolution and widen their scope, so must the analysis tools that bring added value to the raw acquired images. In particular, functional and structural materials, where interfaces play a crucial role, provide important examples where quantification is paramount to microstructure optimization. Electronic packaging, thermal management, smart structures, biomimetic systems, and electrochemistry are typical applications in which the three-dimensional quantification of interfaces carried out by image analysis would be useful.

Here, we use the porous cathode from a Solid Oxide Fuel Cells (SOFC) to demonstrate the utility of an image analysis tool for calculating effective properties (conduction), when taking interface

\footnotetext{
* Corresponding author. Tel.: +334768263 37 .

E-mail addresses: denis.roussel@simap.grenoble-inp.fr (D. Roussel), christophe. martin@simap.grenoble-inp.fr (C.L. Martin).

1 Principal corresponding author.
}

properties into account. Porous cathodes are fabricated through powder processing. Residual porosity (typically 25-50\% [3]) is intentionally kept during sintering to ensure acceptable levels of gas diffusivity. The powder may be either a mixed ion-electron conductor (MIEC) or a mixture of ionic and electronic conducting particles. For the first case, the electrochemical system may be modeled as a homogeneous network of resistances. For the second case, three different resistances must be considered: electronic and ionic resistances for particles in contact with particles of the same type, and electrochemical charge transfer resistance at contacts between two particles of different type. In an SOFC cathode, oxygen is reduced by accepting electrons to form ions:

$\mathrm{O}_{2}+4 e^{-} \rightarrow 2 \mathrm{O}^{2-}$

To facilitate the process, the gaseous oxygen must react where an ionic and an electronic conductor meet. This electrochemical reaction site is known as a triple phase boundary (TPB). The total length of TPBs per unit volume is a good but incomplete measure of cathode performance.

The Finite Element Method (FEM) and Finite Volume Method (FVM) have been used in the literature to compute effective properties of microstructures. Doraswami et al. [4] analyzed anode microstructure using FIB/SEM to compute the total active TPB density and used FEM implemented in COMSOL Multiphysics ${ }^{\mathrm{TM}}$ to 


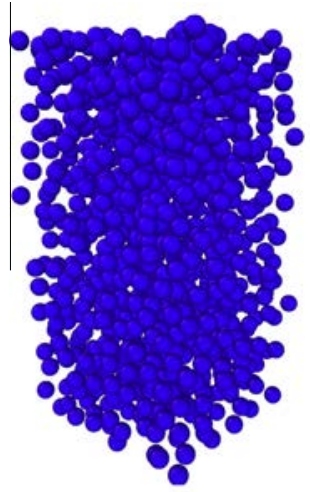

(a)
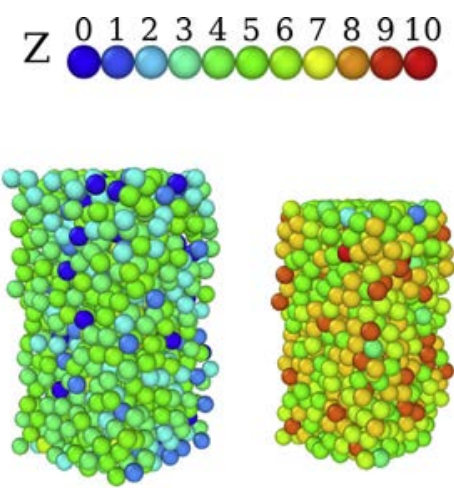

(b)

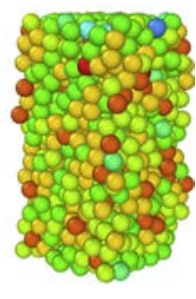

(c)
Fig. 1. Microstructure generated using the Discrete Element Method. (a) Particles are created inside a simulation box without any contact between particles (coordination number $(Z)$ equal to 0 ). (b) They are then packed until a green density of 0.5 is obtained. (c) Finally, the sintering process is simulated, producing overlapping contacts.

predict overpotentials on a 2D model. Liu et al. [5] designed a framework to import, mesh and post-process 3D microstructures allowing non-linear equations to be solved. Kishimoto et al. [6] simulated the power generation performance of an SOFC using FVM and the Bütler-Volmer law. FEM/FVM are powerful methods that are able to solve non-linear system by meshing a volume which can be generated internally [4] or imported from a real microstructure $[5,6]$. The meshing step is critical to acquiring reliable results. A fine meshing will generally give a more precise result but will also increase the computation time. In this paper, a software based on Fast Fourier Transform is used to solve linear equations on real 3D microstructures.

A number of researchers have simulated 3D random mixtures of electronically and ionically conducting particles using numerically generated microstructures [7-10]. Particles are generally modeled as packed spheres in contact with one another. The position of these spheres may be arbitrary $[7,9,10]$ or dictated by realistic mechanical contact laws based on the Discrete Element Method (DEM) [8]. DEM is used to numerically generate particulate microstructures where particles are approximated as spheres. DEM allows for the creation of realistic microstructures by consolidating and then sintering a loose packing of particles. The process has been described in detail in previous papers $[11,12]$ and is schematically summarized in Fig. 1.

These numerical microstructures are useful for understanding the main relations between microstructural parameters (porosity, particle size, particle size distribution, contact size, etc.) and electrochemical performance. Still, they are only a crude representation of real microstructures. The aim of this paper is to present an analysis tool based on ImageJ, a Java-based open source image processing and analysis system [13] which can be used to modify a 3D image to compute its effective conductivity while taking into account charge transfer resistance. The plugin is validated using DEM generated microstructures that have been voxelised. Conduction values are compared to those obtained by the resistance network method.

\section{Methodology}

The effective conductivity is calculated from a simulated porous composite microstructure made of discrete particles. We illustrate the application of the model for the case of an SOFC cathode where both ionic and electronic conductors must be accounted for to compute an effective conductivity. The charge transfer resistance at the contact between ionic and electronic conducting particles is also considered. The effective conductivity depends on the intrinsic conductivity of particles, the charge transfer resistance, the geometry of the contact zone, and the packing of the particles.

Fig. 2 presents the general flow chart for the proposed method. An image is generated using either a DEM numerical microstructure (a) or a real 3D microstructure. The image is treated to replace contacts between particles of different type with a layer representing an electrochemical resistance (b). The effective conductivity is computed using the voxel based software Geodict [14] (c). In parallel, providing that the microstructure has been generated numerically, the effective conductivity can be computed by a resistance network method (d).

The numerical microstructure is generated using 1000 randomly placed particles with an average diameter of $1 \mu \mathrm{m} \pm 5 \%$. This loose packing is compressed to a 0.5 relative density, which is a typical value for green powders before sintering [11]. The relative density $d$ is defined as the ratio between the total volume of particles and the volume of the simulation box:

$d=\frac{\sum_{n_{\text {part }}} V_{\text {part }}}{V_{\text {box }}}$

where $n_{\text {part }}$ is the number of particles, $V_{\text {part }}$ the volume of a particle and $V_{\text {box }}$ the volume of the simulation box. During jamming, contacts between particles appear and at 0.5 relative density, the average contact number per particle is 3.6 (Figs. 1 and 3). The 0.5 relative density is then further increased by simulating the sintering process at high temperature. During sintering, the spherical particles gradually overlap one another, simulating neck formation. Details concerning the sintering procedure can be found in [15]. Coble's model [16] together with volume conservation is used to compute the neck radius $r_{c}$. The same sintering kinetics is assumed for the two materials for simplicity though not by necessity. Sintering is stopped at a relative density of 0.85 . This is because contact impingement may become significant above this threshold, and is not properly taken into account in the numerical sintering stage.

At the start of the sintering process, particles only have few contacts (Fig. 1) and these contacts are not bonded (no overlaps). During the sintering process, the number and the size of contacts increases. The increase in coordination number is depicted in Fig. 3. The contact size normalized with the particle radius $r_{p}$, $\left(r_{c} / r_{p}\right)$ is approximately 0.75 for a relative density of 0.85 . Because particles are initially located randomly within the simulation box, and are rearranged to obtain force equilibrium in the DEM, there exists a distribution of contacts number and a distribution of contact sizes. Fig. 4 shows, for example, the contact size distribution for the two extreme densities studied here (0.5 and 0.8 ).

Conductivity is computed using two different methods. First, taking advantage of the discrete nature of the microstructure, a resistance network is built and an effective conductivity can be calculated from Kirchhoff's current law by applying a potential difference along the direction where the conductivity is computed [8]. With SOFCs, electrochemical reactions occur at the TPBs where the ionic conductor, electronic conductor and gas species meet, resulting in a charge transfer resistance that is accounted for in the resistance network model using a linearized form of the Bütler-Volmer law [8].

The second method consists of voxelizing the numerical microstructure to obtain a 3D thresholded image. This image is then used to compute the conductivity from image analysis using Ohm's law and Fast-Fourier transform [17]. For this case, the charge transfer resistance is accounted for by introducing a resistive layer where the interfaces at active TPB are located. These two methods are detailed in the following section. 


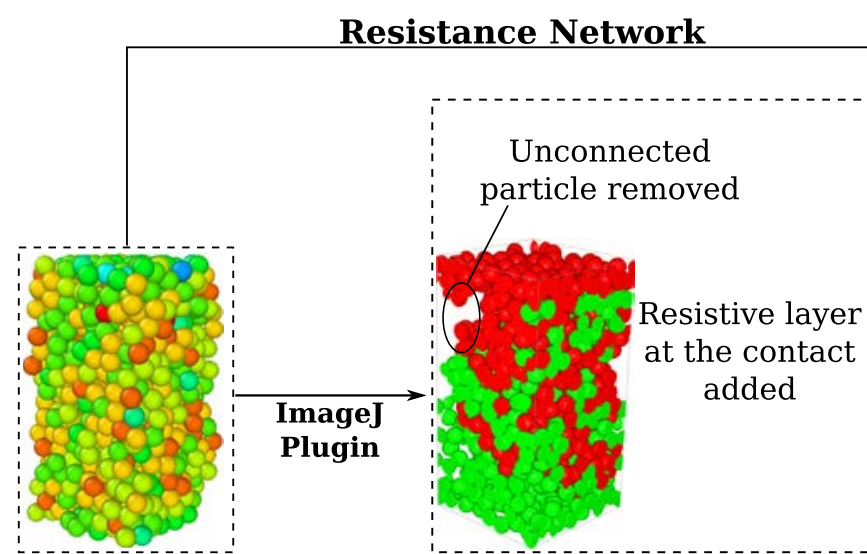

(a) (b)

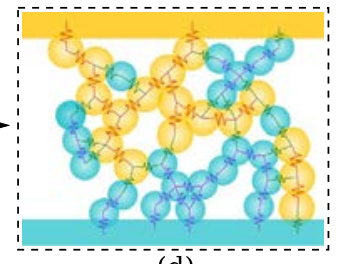

(d)

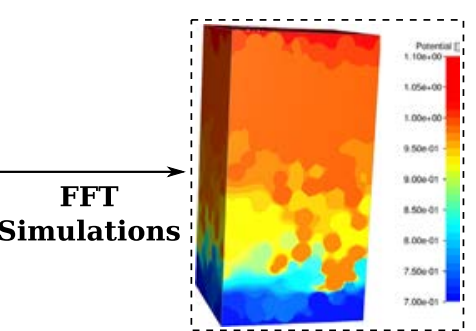

(c)

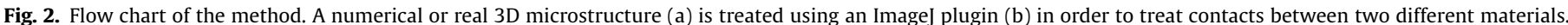

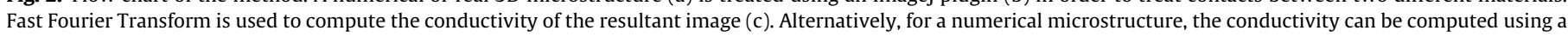
Resistance Network Model (d).

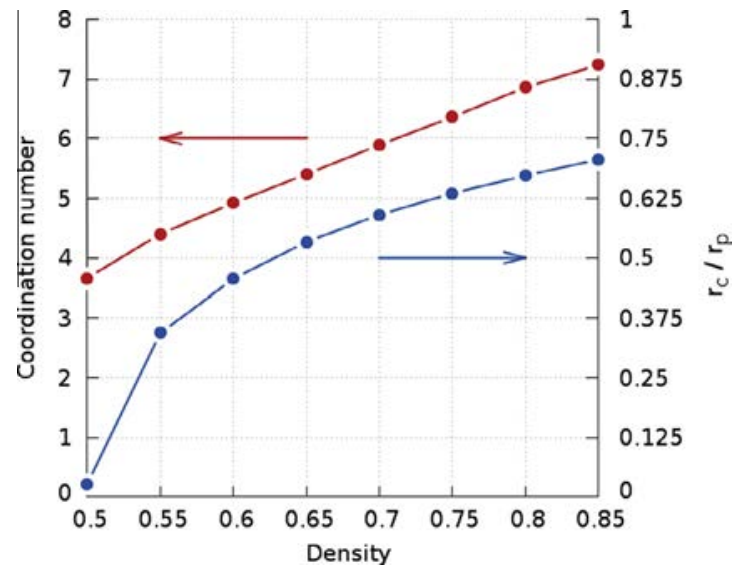

Fig. 3. Evolution of the contact size during the sintering process.

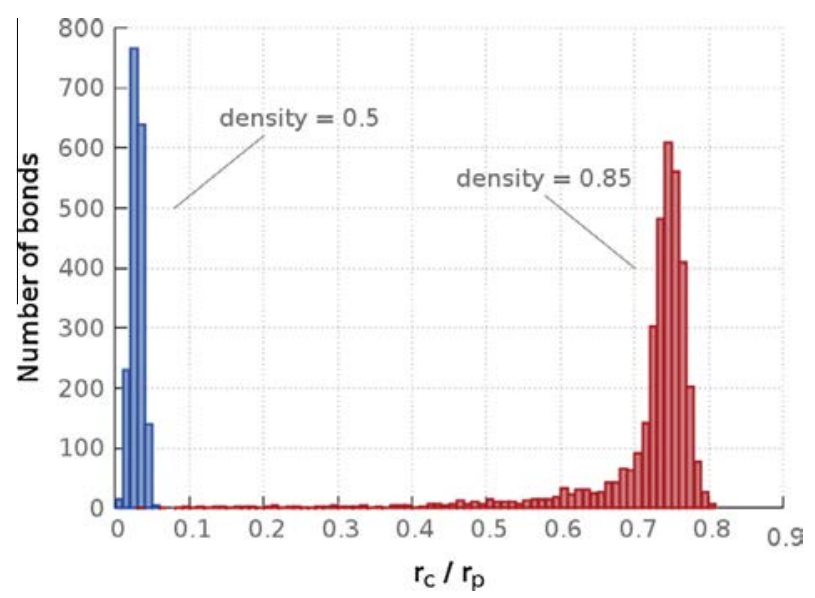

Fig. 4. Distribution of the contact size for the two extreme densities.

\section{Resistance network}

Random resistance networks are a frequently used approach to simulate various types of conductions based on discrete models. Thermal [18] or electronic/ionic $[8,10,19]$ applications in

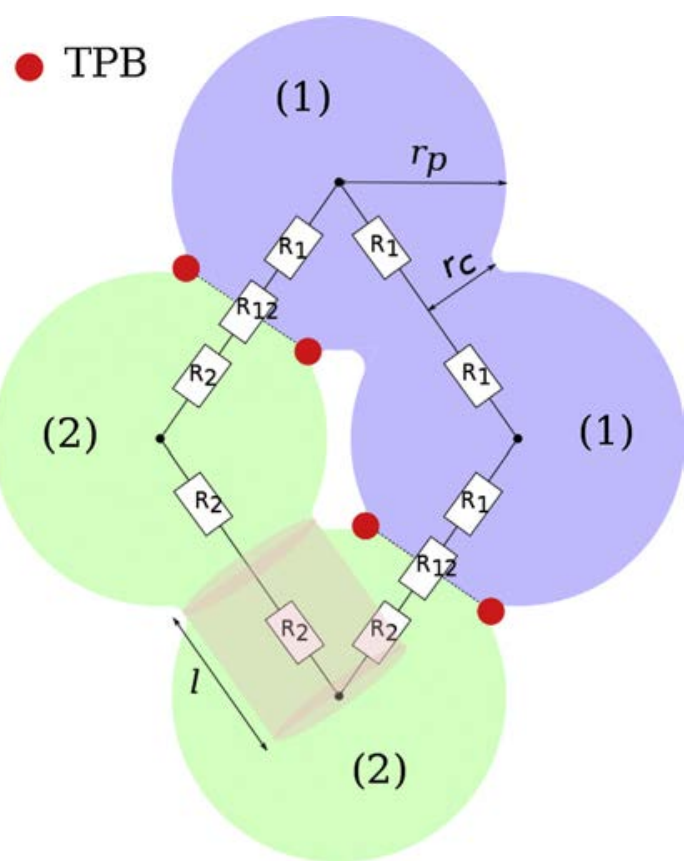

Fig. 5. Schematic of the resistance network model with the two different materials ((1) and (2)) to simulate the microstructure. A contact between two particles of the same type is represented by a single resistance (R1 or R2) for each particle and calculated using Eqs. (2) and (3). A contact between two particles of different type is represented by an additional resistance (R12) to simulate the effect of the charge transfer resistance (Eq. (4)).

particulate media may be computed using this approach. This method consists of replacing contacts between particles by a resistance as shown in Fig. 5.

In this study, electron and ion transport are considered to be ohmic. The resistance of a half sphere $R_{i}$ is calculated using the analytical model of Argento and Bouvard for heat transfer [20]:

$R_{i}=0.899 \times \frac{r_{c}}{r_{p}} \times \frac{R_{i, \mathrm{cyl}}}{\sqrt{1-\left(\frac{r_{c}}{r_{p}}\right)^{2}}}$ for $\frac{r_{c}}{r_{p}}<0.744$

$R_{i}=R_{i, \mathrm{cyl}} \quad$ for $\frac{r_{c}}{r_{p}} \geqslant 0.744$ 
where $r_{p}$ is the particle radius, $r_{c}$ is the contact radius and $R_{i, \text { cyl }}$ is the electrical resistance of a homogeneous cylinder with the same radius as the contact:

$R_{i, \mathrm{cyl}}=\frac{l}{\sigma_{i} \pi r_{c}^{2}}$

with a given intrinsic conductivity $\sigma_{i}$ and length $l$ corresponding to the distance from the center of the particle to its contact point (Fig. 5). The resistance for large contact size $\left(r_{c} / r_{p}>0.744\right)$ is simply approximated by the cylinder resistance to avoid the $R_{i}$ value becoming unrealistically high.

An additional contact resistance between particles of different materials is defined by a resistance that depend on the triple point boundary length $\left(L_{T P B}=2 \pi r_{c}\right)$ and which is due to the kinetics of the charge transfer process [8]:

$R_{\text {inter }}=\frac{1}{\sigma_{\text {inter }}} \times \frac{1}{L_{T P B}}$

where $R_{\text {inter }}$ is the resistance of the interface and $\sigma_{\text {inter }}$ is the intrinsic conductivity of the charge transfer. Eq. (4) is a linearization of the classical Bütler-Volmer equation. This linearization is valid for low current densities [21].

As is typical for SOFC modeling, the particle packing is sandwiched between a current collector and an electrolyte $[8,10]$. This is carried out by assigning electronic or ionic properties to all particles at the top or at the bottom surface, respectively. The current collector and electrolyte are thus very thin (of the order of one particle layer) and assumed to be equipotential. This setup allows the effect of the charge transfer resistance to be taken into account realistically for the SOFC application. Indeed, bypassing this step would result in assigning too large a role to the phase that percolates and which typically has a resistance that is orders of magnitude smaller than the charge transfer resistance $[7,20,22,23]$. Potentials $V_{1}$ and $V_{2}$ are imposed on the current collector and the electrolyte respectively.

A linear system of equations using Kirchhoff's current law is obtained in matrix form:

$[K][V]=[I]$

where $[K]$ is a $N \times N$ matrix of the contact conductances of the $N$ particles in the packing. Vector $[V]$ contains the unknown particle potentials, and the vector $[I]$ contains the total currents in or out of each particle. The effective conductivity of the network is calculated by taking the inverse of the system for the imposed potentials $V_{1}$ and $V_{2}$ and using Ohm's law. The geometry of the electrode is normalized by the ratio $t / S$, where $t$ is the electrode thickness and $S$ is the surface area normal to the thickness:

$\sigma_{\text {eff }}=\frac{I}{V_{1}-V_{2}} \times \frac{t}{S}$

where $I$ is the total current entering or leaving the network. The effective conductivity is in $S \mathrm{~cm}^{-1}$.

\section{Image analysis}

\subsection{Microstructure characteristics}

Numerical microstructures are read as 3D images and analyzed using image analysis software (ImageJ) from which several microstructural properties can be extracted (volume fractions, interfacial area density, connectivity and TPB length).

The composition of a material is generally known before 3D reconstruction. Therefore, the volume fraction of the different phases may be used to verify that the analyzed volume has been segmented correctly and is representative of the larger sample.
Volume fractions are simply calculated by counting the voxels that correspond to each phase.

In a composite material, reactions often occur along interfaces. Thus a larger surface area per unit volume is generally associated with higher activity. The interfacial area density is expressed as the ratio between surface area and the volume of the sample $\left(\mu \mathrm{m}^{-1}\right)$. Simply counting voxels that are located between two phases leads to an overestimation of the interfacial area density due to the voxel discretization. Instead, we use a marching-cube algorithm [24].

For the case of an SOFC cathode, oxygen is reduced into ions at the reaction sites (TPBs). For this to happen, oxygen, which enters laterally in our simulations, must reach the TPBs. TPBs must be connected to the electrolyte through ionic conducting particles and to the current collector through electronic conducting particles. Thus each phase should be connected through a percolating network to a specific location (current collector or electrolyte). Any isolated phase is considered inactive, and is removed from the microstructure (Fig. 2). However, because the high resolution of images from FIB tomography and nano-tomography comes at the expense of relatively small sample volumes, some connected patterns may be seen as unconnected within the small analyzed volume. To account for this uncertainty, phases which are connected to a side that is not an active one are classified as unknown as in $[23,25,26]$.

In order to increase the performance of an SOFC cathode, the TPB density must be maximized. The value of the TPB density typically varies between $2 \mu \mathrm{m}^{-2}$ and $8 \mu \mathrm{m}^{-2}[23,27]$. TPB density is computed using a method presented by Iwai et al. [27]. Each phase is dilated by one voxel and a TPB is found if the three different phases are overlapping. The TPB density is then computed using a marching cube algorithm.

\subsection{Effective conductivity}

The GeoDict software package [17] was used to compute the effective conductivity from our microstructures. GeoDict is based on Fast Fourier Transform and the Bi-CGSTAB method [28]. Each voxel is assigned an intrinsic conductivity and Ohm's law is applied. This law derived from Fourier's law states that the amount of species (i.e. electrons or ions) that flow through a unit area per unit time is proportional to the negative local potential gradient.

$J=-\sigma \nabla \phi$

where $J$ is the current density, $\phi$ the electrical/ionic potential, and $\sigma$ the electrical/ionic conductivity which is a scalar in the case of an isotropic material, as considered here. A potential is imposed in between both side of the microstructure where the conductivity is calculated. Eq. (7) is used to compute the potential of each voxel. The potential on the edge of the sample is calculated using periodic conditions.

We first test the effect of voxel discretization on the effective conductivity. To do so, we use a homogeneous packing (all particles have the same conductivity, and no charge transfer at the interface). Fig. 6 shows the conductivity at increasing resolutions. Simulations have been carried out on six parallel threads with intel Xeon CPUs on a microstructure containing 1000 particles packed to a density of 0.5 and sintered to a density of 0.7 . The resolution of an image is determined by the number of voxels describing the radius of one particle. For example, a simulation box containing 1000 particles with a density of 0.7 voxelised with 500 voxels has a resolution of 28 voxels/particle radius, assuming volume conservation during sintering. With a resolution of 22 voxels/radius, the CPU time is 200s. Using 22 voxels/radius leads to an underestimation of $1 \%$ of the conductivity as compared with the result achieved with 66 voxels/radius which took 20 times longer. 


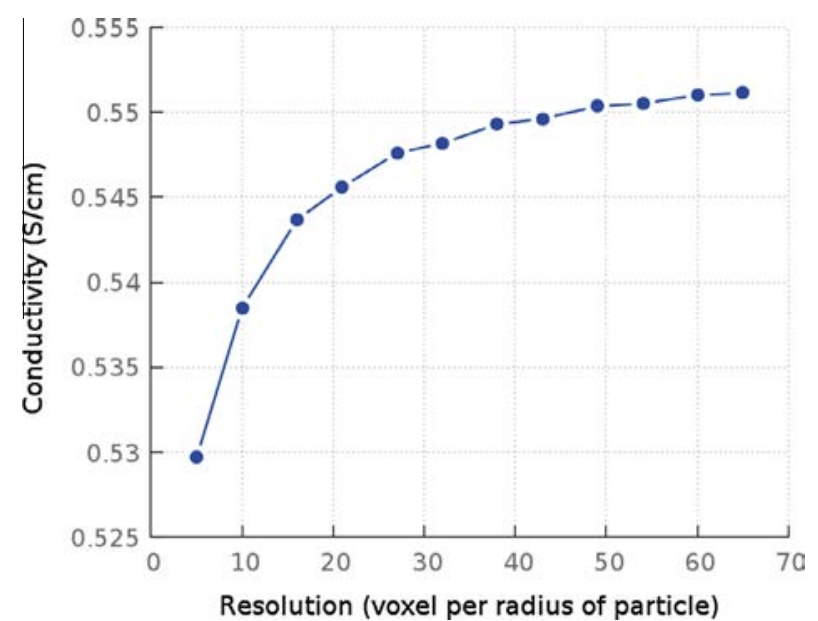

Fig. 6. Evolution of the conductivity for a voxelised microstructure at different resolutions, calculated by FFT.

For composite microstructures, we take into account the charge transfer resistance at interfaces by introducing a resistive layer as shown in Fig. 7. The voxelized interface is defined by any voxel of type 1 (or 2) having a contact with a voxel of type 2 (or 1 ), respectively. Assuming that the thickness of the interface is small and that the area normal to the thickness is constant, the resistance of the layer $R_{\text {layer }}$, can be expressed as a function of the layer conductivity, $\sigma_{\text {layer }}$, the thickness of the layer, $h$, and the area, $A$ :

$R_{\text {layer }}=\frac{1}{\sigma_{\text {layer }}} \times \frac{h}{A}$

When the resistances of the bulk materials are negligible compared to the resistance of the layer, it should be possible to build a resistance network model to compute the effective conductivity. In the more general case however, the FFT model is required to take into account the specificity of the geometry of the structure. By combining Eqs. (4) and (8) we can express the conductivity ratio of the interface layer for the case of an SOFC cathode:

$\frac{\sigma_{\text {layer }}}{\sigma_{\text {inter }}}=\frac{L_{T P B} \cdot h}{A}$

where $L_{T P B}$ is the length of the TPB.

Therefore, we can define a specific conductivity for the layer at the interface that will depend solely on the electrochemical

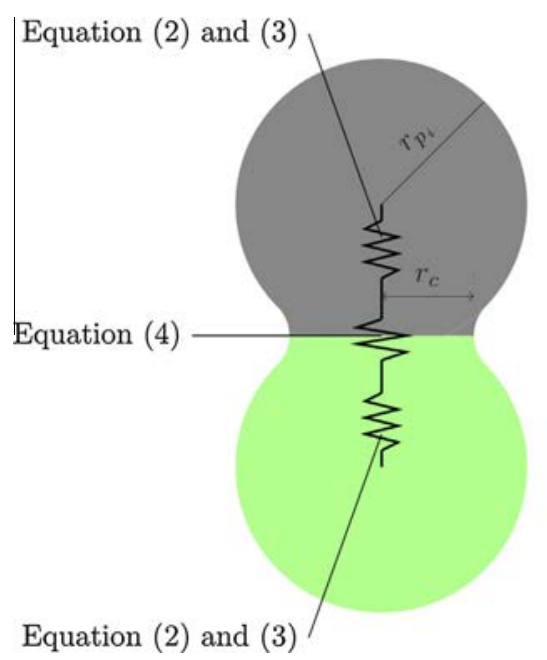

conductivity $\sigma_{\text {inter }}$ and on the geometry of the layer. The result is that the additional layer used here represents the charge transfer resistance needed to represent the effect of the TPB.

ImageJ is used to pretreat the $3 \mathrm{D}$ images before computing the conductivity. A macro defined as 'resistive layer' has been built to introduce the charge transfer resistance into the voxelised volume. It takes a thresholded stack of images containing three phases (pores: material0, electronic conductor: material1 and ionic conductor: material2) as its input and produces a new stack with four phases: three connected materials and one unconnected phase. The three phases are the ionic conducting voxels that are connected to the electrolyte, the electronic conducting voxels that are connected to the current collector and pore voxels that are connected to an external lateral surface. All the other voxels that are left belong to the unconnected phase. Interfaces between material1 and material2 are replaced by a layer with a conductivity, whose value is given by Eq. (9). This value depends on the conductivity of the charge transfer resistance, the length of the TPB, the surface area of the interface and on the thickness of the added layer (Eq. 8). The plugin creates a layer of two voxels at the interface (one voxel for each phase) whatever the resolution of the image. Therefore, to ensure that the contact resistance is resolution independent, the conductivity assigned to a given layer must be adjusted. The algorithm for the plugin is described in [29].

Fig. 7 shows the difference between the two methods used to compute the effective conductivity of a composite material.

\section{Results}

\subsection{Effective conductivity of a homogeneous material}

Discrete simulations (DEM) were used to create different microstructures (Fig. 1) and their conductivities were computed using either a resistance-network model or an image-analysis method, as described in the preceding sections. The two techniques were first compared by computing the normalized effective conductivity for a single material without taking into account the effect of any charge transfer resistance (Fig. 8).

The analytical model of Argento and Bouvard [20] (Eq. (2)), which has been derived for particulate media, fits well with the calculated values derived from image analysis on the voxelised microstructure (Fig. 8), except for the two extreme densities (0.5 and 0.85 , which correspond to $r_{c} / r_{p}=0.02$ and $r_{c} / r_{p}=0.72$, respectively). At 0.85 relative density, Fig. 4 shows that a non-negligible

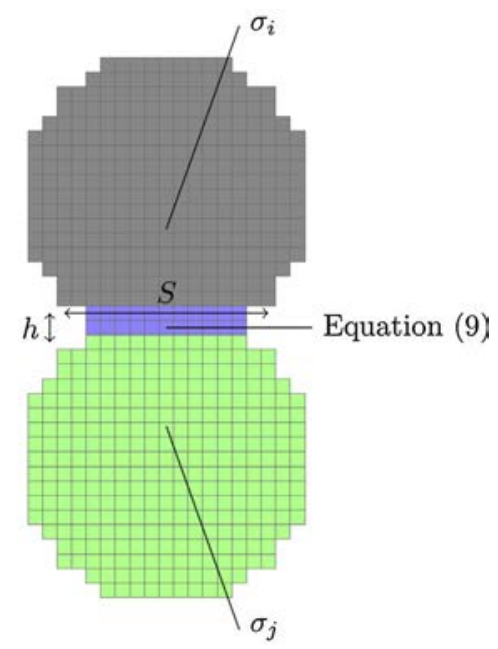

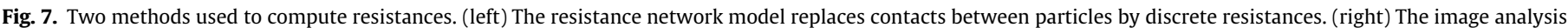

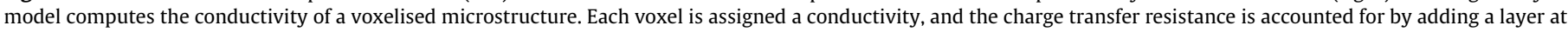
the interface with a conductivity determined by Eq. (9). 


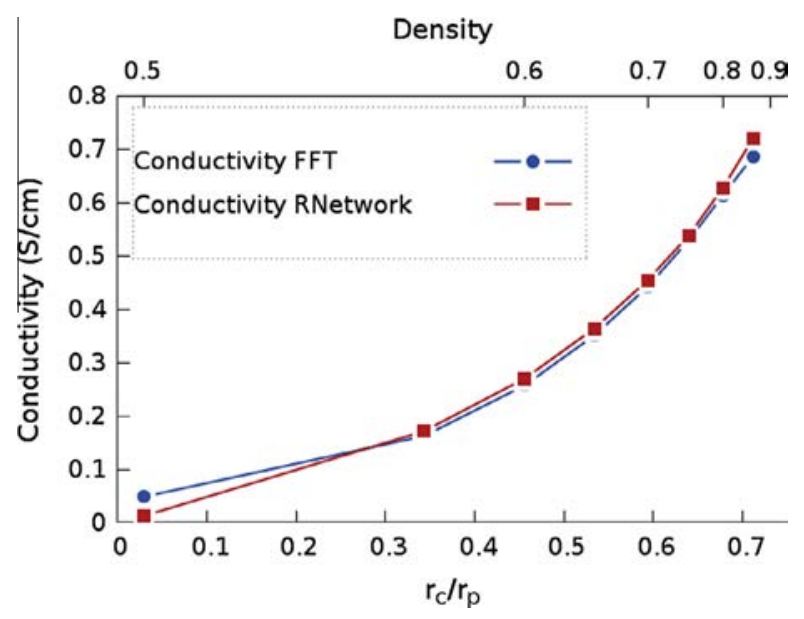

Fig. 8. Comparison of conductivities computed using the 3D image analysis code (FFT) and on a simulated microstructure using the resistance network model which does not take into account charge transfer resistance. The conductivity of the particles is set to $1 \mathrm{~S} \mathrm{~cm}^{-1}$.

number of bonds exhibit a normalized size above 0.744 . Above this threshold, our model simply uses the resistance of a cylinder which overestimates the bond conductivity. Before sintering (density $=0.5$ ), particles have only minute contacts, leading to infinite resistance and to a zero effective conductivity using the resistance network model. The voxelisation poorly captures this initial stage since contact size is overestimated by discrete voxels (a value of 22 voxels/radius has been used).

\subsection{Effective conductivity of a composite microstructure}

Properties of a sintered microstructure with a density of 0.65 are compared using both image analysis and the resistance network method. For the image analysis method, the microstructure is voxelised at different resolutions and the evolution of properties is shown in Fig. 9. Both specific area and TPB density stabilise around 16 voxel per radius. The specific area at this resolution is underestimated by less than $5 \%$ and TPB density is overestimated by less than $5 \%$. Composite microstructures are voxelised at 28 voxel per radius as for the homogeneous material. We have
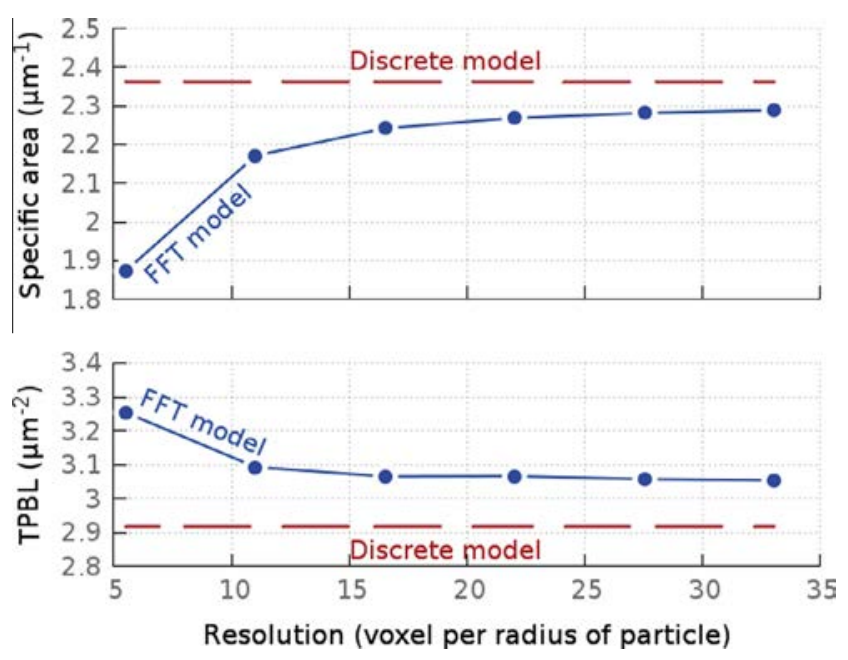

Fig. 9. Comparison of specific area and TPB density for image analysis and resistance network for a microstructure of 1000 particles sintered to a density of 0.65 at different image resolutions.
Table 1

Conductivity parameters.

\begin{tabular}{lll}
\hline Species & Conductivity and corresponding $T$ & Ref \\
\hline Electronic conductor & $200 \mathrm{~S} \mathrm{~cm}^{-1}(800 \mathrm{~K}<T<1200 \mathrm{~K})$ & {$[7]$} \\
Ionic conductor & $0.1 \mathrm{~S} \mathrm{~cm}^{-1}(T=1200 \mathrm{~K})$ & {$[7]$} \\
Charge transfer layer & $1 \mathrm{~d}-5 \mathrm{~S} \mathrm{~cm}^{-1}(T=1200 \mathrm{~K})$ & {$[20,22,23]$} \\
\hline
\end{tabular}

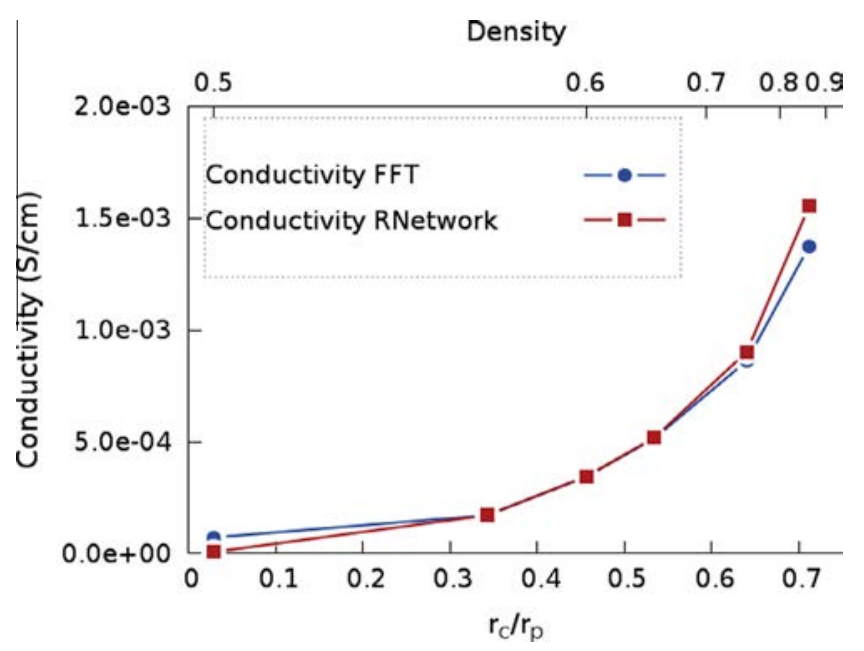

Fig. 10. Comparison of the conductivity computed for a composite material using both the resistance-network method and image analysis.

verified that such a voxel resolution also leads to an error of less than $5 \%$ for dense packing (density $=0.85$ ).

The effective conductivity of a composite SOFC cathode has been calculated using material parameters for the conductivity given in Table 1 . A temperature of $1200 \mathrm{~K}$, a typical to SOFC operating temperature, has been chosen.

The conductivity of a packing of particles, accounting for interface resistance, is shown in Fig. 10 as a function of the normalized contact size $\left(r_{c} / r_{p}\right)$. As the size of contacts increases, the density of the particle packing increases (second axis in Fig. 10). The conductivity calculated with the resistance-network model is compared to image analysis (FFT). The two methods compare reasonably well, thus validating the overall approach. As for the calculation without charge transfer resistance (Fig. 8), the FFT method leads to larger values that the resistance network method for small contact values. In this case, the network model, which better describes the small contact resistances (Argento-Bouvard model) between two slightly interpenetrating spheres, is better adapted. The FFT method, which relies on the voxelization of the packing, does not allow for a precise description of very small contacts. Conversely, for large contact sizes, the network model leads to a larger effective conductivity than FFT. This is because for the largest average contact size $\left(r_{c} / r_{p}=0.72\right)$, the resistance of large contacts $\left(r_{c} / r_{p}>0.744\right)$ in the packing is simply given by the equivalent cylinder resistance (Eq. (3)) which underestimates the resistance value. However, overall the results show that by replacing TPBs with a resistive layer at the interface between the two different conductors is in good agreement with the linearized form of the Bütler-Volmer law. The results of the two methods follow a similar trend to the one seen for a homogeneous material as shown in Fig. 10.

The method is versatile enough to be applied to other problems where interfaces play a role. The number of materials could also be extended. A three phases sample $(1,2,3)$ exhibits four different interfaces $(1-2,1-3,2-3,1-2-3)$ whereas a four phases material will exhibit 10 interfaces which must be determined. Although 
doable, this extension will require a clear understanding of the various physics affecting each interface.

\section{Conclusion}

A method, based on image analysis coupled with FFT calculation, has been developed to compute an effective conductivity from a voxelised microstructure by substituting the interface between two different materials with a layer having properties that mimic charge transfer. The method has been validated on a homogeneous material (no interface) and on a composite material (with interfaces) by comparing the results with a resistance network model. Providing a sufficient resolution is used (22 voxels/radius for spherical particles) and sufficiently large contacts are modeled $\left(r_{c} / r_{p}>0.1\right)$, the developed method allows an effective conductivity to be computed. The advantage of the method based on image analysis is that it provides a tool to analyze tomography images that come from real microstructures. An ImageJ plugin has been built to add an interface layer between particles of different materials with conductivity as a function of the TPB length and TPB surface area [29].

\section{Acknowledgements}

The Agence Nationale pour la Recherche (ANR) and the National Science Foundation are greatly acknowledged for their financial support (OPTIMA_SOFC project and NSF-DMR Grant \# 1008600).

\section{References}

[1] Z. Yan, O. Guillon, C.L. Martin, S. Wang, C.-S. Lee, D. Bouvard, Appl. Phys. Lett 102 (22) (2013) 31-38.

[2] Y.S. Chu, J.M. Yi, F. De Carlo, O Shen, W.-K. Lee, H.J. Wu, C.L. Wang J.Y. Wang, C.J. Liu, C.H. Wang, S.R. Wu, C.C. Chien, Y. Hwu, A. Tkachuk, W. Yun, M. Feser K.S. Liang, C.S. Yang, J.H. Je, G. Margaritondo, Appl. Phys. Lett. 92 (10) (2008).

[3] S.M. Haile, Acta Mater. 51 (19) (2003) 5981-6000. the Golden Jubilee Issue. Selected topics in Materials Science and Engineering: Past, Present and Future.
[4] U. Doraswami, P. Shearing, N. Droushiotis, K. Li, N. Brandon, G. Kelsall, Solid State Ionics 192 (1) (2011) 494-500. Proceedings of the 17th International Conference on Solid State Ionics.

[5] M.E. Lynch, D. Ding, W.M. Harris, J.J. Lombardo, G.J. Nelson, W.K. Chiu, M. Liu, Nano Energy 2 (1) (2013) 105-115.

[6] M. Kishimoto, H. Iwai, M. Saito, H. Yoshida, J. Power Sour. 196 (10) (2011) 4555-4563.

[7] S. Sunde, J. Electrochem. Soc. 143 (1996) 1930-1939.

[8] L. Schneider, C. Martin, Y. Bultel, D. Bouvard, E. Siebert, Electrochim. Acta 52 (1) (2006) 314-324.

[9] J. Golbert, C.S. Adjiman, N.P. Brandon, Indust. Eng. Chem. Res. 47 (20) (2008) $7693-7699$.

[10] A. Abbaspour, J.-L. Luo, K. Nandakumar, Electrochim. Acta 55 (12) (2010) 3944-3950.

[11] C.L. Martin, R.K. Bordia, Phys. Rev. E 77 (2008) 031307.

[12] X. Liu, C.L. Martin, G. Delette, J. Laurencin, D. Bouvard, T. Delahaye, J. Power Sour. 196 (4) (2011) 2046-2054.

[13] C.A. Schneider, W.S. Rasband, K.W. Eliceiri, Nature Meth. 9 (7) (2012) 675

[14] http://www.math2market.de/.

[15] C.L. Martin, D. Bouvard, G. Delette, J. Am. Ceram. Soc. 89 (11) (2006) 33793387.

[16] R.L. Coble, J. Am. Ceram. Soc. 41 (2) (1958) 55-62.

[17] A. Wiegman, A. Zemitis, Ej-heat: A Fast Explicit Jump Harmonic Averaging Solver for the Effective Heat Conductivity of Composite Materials, Tech. rep., Fraunhofer, 2006.

[18] Z. Chen, X. Luo, S. Liu, Thermal analysis of 3d packaging with a simplified thermal resistance network model and finite element simulation, in: 11th International Conference on Electronic Packaging Technology High Density Packaging (ICEPT-HDP), 2010, 2010, pp. 737-741.

[19] A. Ali, X. Wen, K. Nandakumar, J. Luo, K.T. Chuang, J. Power Sour. 185 (2) (2008) 961-966.

[20] C. Argento, D. Bouvard, Int. J. Heat Mass Transfer 39 (7) (1996) 1343-1350.

[21] A.V. Virkar, J. Chen, C.W. Tanner, J.-W. Kim, Solid State Ionics 131 (12) (2000) 189-198.

[22] R. Radhakrishnan, A.V. Virkar, S.C. Singhal, J. Electrochem. Soc. 152 (1) (2005) A210-A218.

[23] J.R. Wilson, A.T. Duong, M. Gameiro, H.-Y. Chen, K. Thornton, D.R. Mumm, S.A. Barnett, Electrochem. Commun. 11 (5) (2009) 1052-1056.

[24] W.E. Lorensen, H.E. Cline, SIGGRAPH Comput. Graph. 21 (4) (1987) 163-169.

[25] J.S. Cronin, J.R. Wilson, S.A. Barnett, J. Power Sour. 196 (5) (2011) 2640-2643.

[26] D. Chen, Z. Lin, H. Zhu, R.J. Kee, J. Power Sour. 191 (2) (2009) 240-252.

[27] H. Iwai, N. Shikazono, T. Matsui, H. Teshima, M. Kishimoto, R. Kishida, D. Hayashi, K. Matsuzaki, D. Kanno, M. Saito, H. Muroyama, K. Eguchi, N. Kasagi, H. Yoshida, J. Power Sour. 195 (4) (2010) 955-961.

[28] H. van der Vorst, SIAM J. Scient. Statist. Comput. 13 (2) (1992) 631-644.

[29] http://imagejdocu.tudor.lu/doku.php?id=plugin:3d:sort_contact:start. 